\title{
The expression of Programmed death ligand 2 in patients with thymoma and thymomatous Myasthenia Gravis
}

\author{
Haoshuai Zhu ${ }^{1}$, xin zhang ${ }^{1}$, Yanfeng Wang ${ }^{1}$, Su Chen ${ }^{1}$, Tingfei Chen ${ }^{1}$, Bo Zeng ${ }^{1}$, Jianyong \\ $\mathrm{Zou}^{1}$, and Chunhua $\mathrm{Su}^{1}$ \\ ${ }^{1}$ Sun Yat-sen University First Affiliated Hospital
}

May 11, 2020

\begin{abstract}
Abstract Background There is a growing relationship between PD-1/PD-Ls and autoimmune disease We attempted to explore the relationship of the PD-L2 expression in patients with thymoma, especially with Myasthenia Gravis (thymomatous MG). Methods Seventy patients with thymoma who underwent surgical resection between January 2017 to December 2018 were retrospectively reviewed. The PD-L2 expression was evaluated by immunohistochemistry. The association between the PD-L2 expression and the clinicopathologic features was investigated, especially thymomatous MG. Results The PD-L2 expression was positive in 41 patients (58.6\%) and negative in 29 patients (41.4\%). The PD-L2 expression was significantly associated with WHO histology of type B2 and B3 thymoma $(\mathrm{p}=0.008)$ and the status of MG $(\mathrm{p}=0.002)$. In addition, PD-L2 positive tumors showed a significantly smaller tumor size $(\mathrm{P}=0.017)$. Ectopic thymus was significantly more often seen in the $\mathrm{PD}-\mathrm{L} 2$ positive group $(\mathrm{p}=0.035)$. The patients with MG $(\mathrm{p}=0.001)$ and WHO type B2 and B3 $(\mathrm{p}=0.007)$ have significantly higher PD-L2 scores. Multivariate logistic regression model showed the status of MG (OR 12.601, 95\%CI 2.406-65.995, p=0.02) and age (OR $0.199,95 \%$ CI $0.046-0.863, \mathrm{p}=0.031$ ) were significantly associated with the expression of PD-L2. The analysis of 33 patients with MG shows age was not associated with the expression of PD-L2 (OR 0.04, 95\%CI 0.001-3.2, p=0.15). Conclusions A strong expression of PD-L2 in thymoma was significantly associated with thymomatous MG and WHO histologic type B2 and B3. In addition, PD-L2 may play a potential role in the pathogenesis of thymomatous MG.
\end{abstract}

\section{Introduction}

Thymoma is the most common anterior mediastinal mass in adult ${ }^{[1]}$. About $30 \%$ of patients with thymoma have MG (thymomatous MG) ${ }^{[2]}$. Almost all patients with thymomatous MG have antibodies to ACHR ${ }^{[3]}$. However, the pathogenesis of thymomatous MG and the signal path of antibodies generation are not entirely clear. Studies reveal that TFH cells play a fundamental role in humoral immunity deriving from their ability to provide help for germinal center (GC) formation, B cell differentiation into plasma cells and memory cells, and antibody production in secondary lymphoid tissues ${ }^{[4]}$. Furthermore, Lieping Chen and his colleagues found that PD-1 expression on T cells and PD-L2 expression on B cells controlled TFH and PC numbers. PD-1 regulates germinal center B cell survival and the formation and affinity of long-lived plasma cell ${ }^{[5]}$.

What's more, thymic TFH cells might involve in the pathogenesis of MG with thymoma ${ }^{[6,7]}$.

PD-1 is highly expressed in TFH. The roles of PD-1/PD-L1,2 signaling in the pathogenesis of thymomatous MG has been virtually unstudied. However, numerous studies showed that PD-L1 protein expression was not associates with the status of $M G^{[8,9]}$. In that case, PD-L2 may be an alternative and valuable marker. Previous studies shown that PD-L2 was expressed on solid tumor, APC (Regulation of PD-1, PD-L1, and PDL2 expression during normal and autoimmune responses) and medullary thymic epithelial cells ${ }^{[10]}$ with high expression in some organs ( like lung, liver and heart) and low expression in some others (like spleen, lymph 
node and thymus. And there is a growing relationship between PD-1/PD-Ls and autoimmune disease ${ }^{[11]}$. So, we have a guess that PD-L2 may play an important role in the status of MG in patients with thymoma.

The purposes of this retrospective study were to characterize the association between the expression of PDL2 in thymoma and the clinicopathologic features of the patients and to evaluate the relationship of PD-L2 expression and the status of MG.

\section{Patients and methods}

This study was a retrospective review of patients with thymoma and was approved by the Institutional Review Board in the first affiliated hospital of SAN-YAT-SUN university. Seventy patients who had undergone surgical resection for thymomas at the first affiliated hospital of SAN-YAT-SUN university between January 2017 to December 2018 were enrolled, and their paraffin-embedded specimens were used to assess the expression of PD-L2 by immunohistochemistry (IHC). Clinical data were obtained from patients' medical charts, including patient age, sex, tumor size, World Health Organization histologic type, status of myasthenia gravis, Masaoka-Koga stage, ectopic thymus. For the patients with MG, Myasthenia Gravis Foundation of America (MGFA) Clinical Classification at diagnosis were obtained.

Immunohistochemistry Studies Immunohistochemistry studies were performed on formalin-fixed, paraffinembedded tissue sections from each tumor. Each section was cut to a 4-mm thickness. Anti-PD-L2 mouse immunoglobulin G monoclonal antibody (clone UMAB223; ZSGB Bioscience) were diluted to 1:100 and used as a primary antibody. The IHC staining was performed automatically with the Ventana BenchMark XT Stainer (Roche Diagnostics, Basel, Switzerland).

Scoring of PD-L2 Positivity Two pathologists evaluated the expression of PD-L2 on tumor cells. They reviewed each sample independently then discussed the findings of each sample and determined the proportion of PD-L2 positive tumor cells together. According to a previous consensus, a tumor cell was defined as "PDL2 positive" when the cell membranes were partially or completely stained ${ }^{[12]}$ (Fig 1). In contrast, PD-L1 staining in the cytoplasm of a tumor cell was defined as "negative." The PD-L2 positive immune cells, such as lymphocytes and macrophages, were excluded from the cell counts. Tumor cells were quantified by evaluating the ratio of stained to unstained tumor cells. The PD-L2 positivity was evaluated based on based on the proportion of PD-L2 positive tumor cells. A PD-L2 expression rate of $1 \%$ or greater was defined as PD-L2 positive, and all other cases were PD-L2 negative in the present study. The intensity of PD-L2 (from 0 to 3 : 0 , negative; 1 , very weak; 2 , moderate; and 3 , strong) in tumor cells were evaluated for each core. And mean PD-L2 expression scores were calculated by multiplying the percentage of tumor area stained by the staining intensity.

Descriptive statistics were used to describe the patients' baseline. Univariate analysis was conducted using non-parametric test, the Chi square $\left(\chi^{2}\right)$ test and the Student t-test. If necessary, fisher's exact test was conducted for the categorical variables. The logistic regression model was used for the analysis of the correlation between patients' characteristics and PD-L2 expression. The p values of $<0.05$ were defined as significant. 


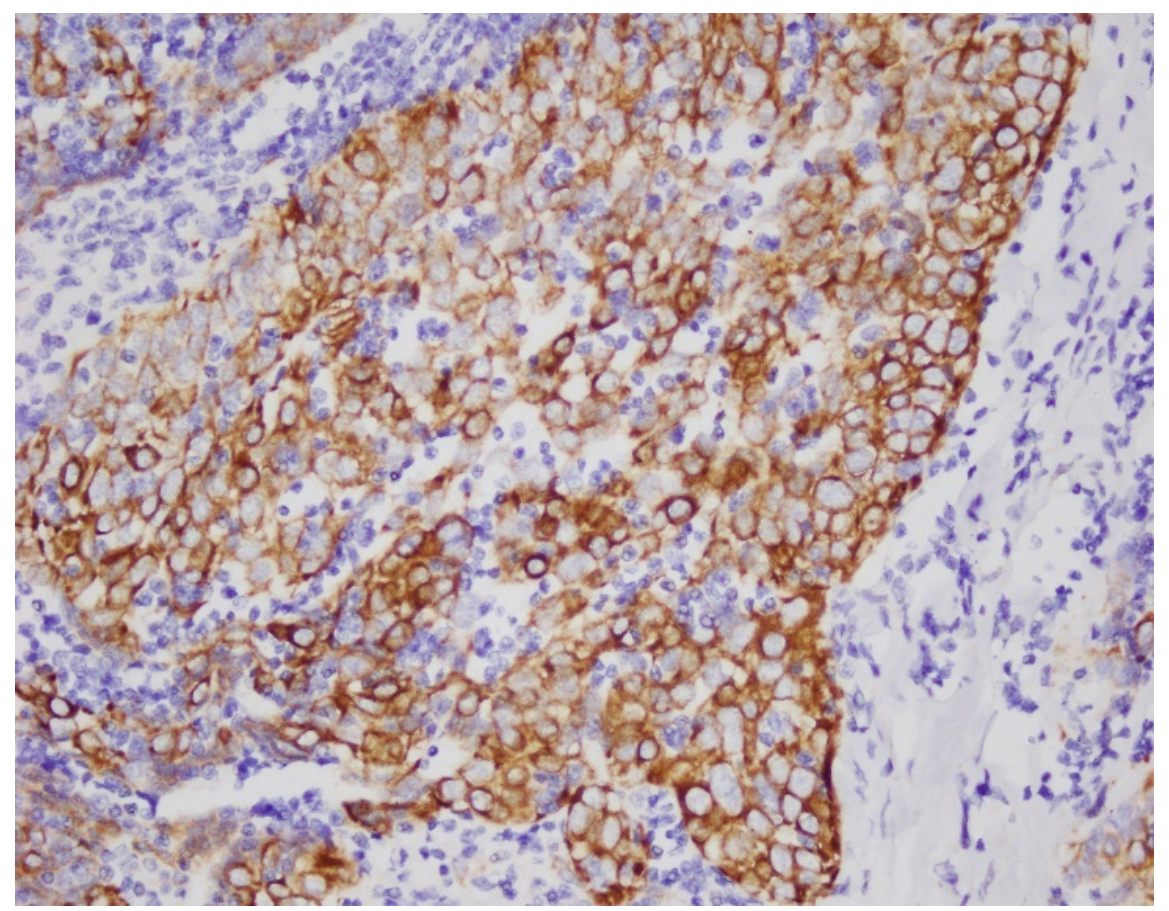

Fig 1. Programmed death ligand 2 (PD-L2) immunohistochemical staining in thymoma. In this case, the tumor cells had stained cell membranes, and this tumor was defined as PD-L2 positive. The proportion of PD-L1 positive tumor cells was $95 \%$ and staining intensity score 3 .

(magnification, $\times 200$ ).

\section{Results}

1, clinicopathologic characteristics

There were 70 patients enrolled in this study. As list in Table 1, the PD-L2 expression was positive in 41 patients $(58.6 \%)$ and negative in 29 patients $(41.4 \%)$. A positive expression was shown in Fig 1 . The mean PD-L2 expression scores was 70.8. Table 1 shows the clinicopathologic characteristics of these patients. The mean age was $49.60 \%$ of patients were male. And the mean tumor size was $5.35 \mathrm{~cm}$. B1 $(17.1 \%)$ and B2 $(44.3 \%)$ dominated the WHO pathologic type. Most of the patients were diagnosed with Masaoka-Koga stage I (71.4\%). 33 patients (47.1\%) were also MG patients.

\begin{tabular}{llll}
\hline Data & Data & No. of patients & percentage \\
\hline Gender & & & \\
& male & 42 & $60 \%$ \\
& female & 28 & $40 \%$ \\
Age & & $49.0(18-76)$ & \\
Tumor size(cm) & & $5.35(1-15)$ & \\
Masaoka-Koga stage & & 50 & $71.40 \%$ \\
& I & 11 & $15.70 \%$ \\
& IIA & 1 & $1.40 \%$ \\
& IIB & 3 & $4.30 \%$ \\
& IIIA & 3 & $2.30 \%$ \\
& IIIB & 2 & $2.90 \%$
\end{tabular}




\begin{tabular}{|c|c|c|c|}
\hline Data & Data & No. of patients & percentage \\
\hline \multicolumn{4}{|c|}{ WHO type } \\
\hline & A & 8 & $11.40 \%$ \\
\hline & $\mathrm{AB}$ & 10 & $14.30 \%$ \\
\hline & B1 & 12 & $17.10 \%$ \\
\hline & B2 & 31 & $44.30 \%$ \\
\hline & B3 & 9 & $12.90 \%$ \\
\hline \multicolumn{4}{|c|}{ Myasthenia gravis } \\
\hline & negative & 37 & $52.90 \%$ \\
\hline & positive & 33 & $47.10 \%$ \\
\hline & MGFA class & & \\
\hline & I & 10 & $14.30 \%$ \\
\hline & IIA & 4 & $5.70 \%$ \\
\hline & IIB & 17 & $24.30 \%$ \\
\hline & IIIA & 1 & $1.40 \%$ \\
\hline & IIIB & 1 & $1.40 \%$ \\
\hline \multicolumn{4}{|c|}{ PDL2 expression } \\
\hline & negative & 29 & $41.40 \%$ \\
\hline & positive & 41 & $58.60 \%$ \\
\hline & PDL2 expression intensity & & \\
\hline & 0 & 29 & $41.40 \%$ \\
\hline & 1 & 16 & $22.90 \%$ \\
\hline & 2 & 11 & $15.70 \%$ \\
\hline & 3 & 14 & $20 \%$ \\
\hline & PDL2 expression ratio & $31.9+30.1 \%$ & $0-98 \%$ \\
\hline & PDL2 scores & $70.8+102.7$ & $0-294$ \\
\hline
\end{tabular}

Table 1 clinicopathologic characteristics and PD-L2 expression in 70 patients

2, correlation between PD-L2 expression and clinicopathologic characteristics

Table 2 shows the clinicopathologic characteristics stratified by PD-L2 and PD-L2 scores stratified by clinicopathologic characteristics. The PD-L2 expression was significantly associated WHO histology of type B2 and B3 thymoma $(\mathrm{p}=0.008)$. And the PD-L2 scores were significantly higher in patients with WHO type B2 and B3 (98.9 vs 33.4, $\mathrm{p}=0.007$ ). The PD-L2 positive group also had a significantly higher proportion of the status of MG than without MG ( $\mathrm{p}=0.002)$. The PD-L2 scores were significantly higher in MG positive group (124.1 vs 23.3, $\mathrm{p}=0.001$ ). In addition, PD-L2 positive tumors showed a significantly smaller tumor size than the PD-L2 negative group $(\mathrm{P}=0.017)$. Ectopic thymus has been significantly more often seen in the PD-L2 positive group than the negative group $(\mathrm{p}=0.035)$. However, no significantly difference considering PD-L2 expression was observed in the age $(\mathrm{p}=0.275)$, gender $(\mathrm{p}=0.621)$ or Masaoka-Koga stage $(\mathrm{p}=0.791)$. The PD-L2 scores were not significantly different in gender $(\mathrm{p}=0.736)$, age $(\mathrm{p}=0.23)$, Masaoka-Koga stage $(\mathrm{p}=0.26)$, tumor size $(\mathrm{p}=0.111)$ or ectopic thymus $(\mathrm{p}=0.115)$. We conducted a logistic regression model to investigate PD-L2 expression (Table 3). The status of MG (OR 12.601, 95\%CI 2.406-65.995, $\mathrm{p}=0.02$ ) and age (OR 0.199, 95\% CI 0.046-0.863, $\mathrm{p}=0.031$ ) were significantly associated with the expression of PD-L2. WHO histology type, Masaoka-Koga stage, tumor size or ectopic thymus were not associated with PD-L2 expression.

\begin{tabular}{|c|c|c|c|c|c|c|}
\hline Data & Data & PDL2 positive & PDL2 negative & $\mathrm{p}$ & PD-L2 scores & $\mathrm{p}$ \\
\hline \multirow[t]{2}{*}{ Gender } & male & 26 & 16 & 0.621 & $74.2 \pm 108.2$ & 0.736 \\
\hline & female & 15 & 13 & & $66.7 \pm 95.6$ & \\
\hline Age(years) & Age(years) & $50.5 \pm 12.6$ & $47.0 \pm 13.5$ & 0.275 & $85.2 \pm 109.3$ & 0.230 \\
\hline
\end{tabular}




\begin{tabular}{|c|c|c|c|c|c|c|c|}
\hline Data & Data & PDL2 positive & PDL2 negative & $\mathrm{p}$ & & PD-L2 scores & $\mathrm{p}$ \\
\hline \multirow{3}{*}{ Myasthenia gravis } & & & & & $>49$ & $55.6 \pm 94.5$ & \\
\hline & positive & 26 & 7 & 0.002 & & $124.1 \pm 117.3$ & 0.001 \\
\hline & negative & 15 & 22 & & & $23.3 \pm 55.5$ & \\
\hline \multirow[t]{2}{*}{ WHO type } & $\mathrm{A}+\mathrm{AB}+\mathrm{B} 1$ & 18 & 12 & 0.008 & & $33.4 \pm 72.1$ & 0.007 \\
\hline & $\mathrm{B} 2+\mathrm{B} 3$ & 29 & 11 & & & $98.9 \pm 113.7$ & \\
\hline \multirow[t]{2}{*}{ Masaoka-Koga stage } & I & 30 & 20 & 0.791 & & $79.6 \pm 108.5$ & 0.260 \\
\hline & IIA-IV & 11 & 9 & & & $48.8 \pm 85.4$ & \\
\hline \multirow[t]{2}{*}{ Tumor size $(\mathrm{cm})$} & Tumor size $(\mathrm{cm})$ & $4.7 \pm 2.4$ & $6.2 \pm 2.8$ & 0.017 & {$[?] 5 \mathrm{~cm}$} & $89.7 \pm 114.2$ & 0.111 \\
\hline & & & & & $>5 \mathrm{~cm}$ & $50.8 \pm 86.2$ & \\
\hline \multirow[t]{2}{*}{ Ectopic thymus } & yes & 11 & 2 & 0.035 & & $111.4 \pm 124.4$ & 0.115 \\
\hline & no & 30 & 27 & & & $61.5 \pm 96.0$ & \\
\hline
\end{tabular}

Table 2 correlation between PD-L2 expression and clinicopathologic characteristics

\begin{tabular}{lllll}
\hline Data & & OR & $95 \% \mathrm{CI}$ & $\mathrm{p}$ \\
\hline Gender & male & 1.972 & $0.569-6.836$ & 0.284 \\
female & 1 & & \\
Age(years) & {$[?] 49$} & 0.199 & $0.046-0.863$ & 0.031 \\
Myasthenia gravis & $>49$ & 1 & & \\
& $\begin{array}{l}\text { positive } \\
\text { negative }\end{array}$ & 12.601 & $2.406-65.995$ & 0.003 \\
WHO type & A+AB+B1 & 0.407 & $0.115-1.437$ & 0.163 \\
Masaoka-Koga stage & B2+B3 & 1 & & \\
I & IIA-IV & 1.728 & $0.414-7.211$ & 0.453 \\
Tumor size & {$[?] 5 \mathrm{~cm}$} & 0.594 & $0.156-2.263$ & 0.446 \\
Ectopic thymus & $\begin{array}{l}\text { yes } \\
\text { yo }\end{array}$ & 1 & & \\
& no & 1 & & \\
\hline
\end{tabular}

Table 3 Multivariate Logistic regression analysis of PD-L2 expression by clinicopathologic characteristics

3, MG and PD-L2 expression, clinicopathologic characteristics

Table 4 shows the compare of clinicopathologic characteristics between MG (+) group and MG (-) group. The patients with WHO histology of type B2 and B3 have a significantly higher incidence rate of MG $(\mathrm{p}=0.004)$. The patients with MG have smaller tumor size $(\mathrm{p}<0.001)$ and higher PD-L2 scores $(\mathrm{p}<0.001)$. No significantly difference were observed in age $(\mathrm{p}=0.052)$, gender $(\mathrm{p}=0.224)$, Masaoka-Koga stage $(\mathrm{p}=1)$ or ectopic thymus $(\mathrm{p}=0.357)$. Due to the positive association between MG and PD-L2 expression, we particularly analyzed the data of patients with MG to explore if the PD-L2 expression depended on the different features of MG (Table 5). 33 patients with MG were enrolled. There were no significantly difference of PD-L2 scores regarding gender, age, MGFA stage, WHO histology type, Masaoka-Koga stage, tumor size or ectopic thymus in patients with MG. We conducted a logistic regression model to predict PD-L2 expression in patients with MG. No feature listed above was associated with PD-L2 expression in patients with MG.

\begin{tabular}{lllll}
\hline Data & Data & MG positive & MG negative & $\mathrm{p}$ \\
\hline Gender & male & 17 & 25 & 0.224 \\
& female & 16 & 12 &
\end{tabular}




\begin{tabular}{lllll}
\hline Data & Data & MG positive & MG negative & $\mathrm{p}$ \\
\hline Age & & $45.8 \pm 12.8$ & $51.9 \pm 12.7$ & 0.052 \\
WHO type & A+AB+B1 & 8 & 22 & 0.004 \\
& B2+B3 & 25 & 15 & \\
Masaoka-Koga & I & 24 & 26 & 1 \\
& IIA-IV & 9 & 11 & \\
Tumor size & & $4.1 \pm 2.3$ & $6.5 \pm 2.4$ & 0.001 \\
Ectopic thymus & yes & 8 & 5 & 0.357 \\
& no & 25 & 32 & \\
\hline
\end{tabular}

Table 4 correlation between MG clinicopathologic characteristics

\begin{tabular}{|c|c|c|c|c|c|c|}
\hline Data & Data & No. of patients & PD-L2 scores & PD-L2 scores & PD-L2 expression & PD-L2 expressic \\
\hline & & & PD-L2 scores & $\mathrm{p}$ & OR & $95 \%$ CI \\
\hline \multirow[t]{2}{*}{ Gender } & male & 17 & $141.0 \pm 125.7$ & 0.402 & 2.488 & $0.234-26.444$ \\
\hline & female & 16 & $106.1 \pm 108.8$ & & 1 & \\
\hline \multirow[t]{2}{*}{ Age(years) } & {$[?] 49$} & 22 & $123.8 \pm 116.4$ & 0.982 & 0.04 & $0.001-3.2$ \\
\hline & $>49$ & 9 & $124.8 \pm 125.7$ & & 1 & \\
\hline \multirow[t]{2}{*}{ MGFA class } & $\mathrm{I}+\mathrm{IIA}$ & 13 & $121.6 \pm 127.4$ & 0.92 & 0.151 & $0.011-2.009$ \\
\hline & IIB-IV & 18 & $125.9 \pm 112.8$ & & 1 & \\
\hline \multirow[t]{2}{*}{ WHO type } & $\mathrm{A}+\mathrm{AB}+\mathrm{B} 1$ & 8 & $61.6 \pm 92.3$ & 0.061 & 0.198 & $0.016-2.514$ \\
\hline & $\mathrm{B} 2+\mathrm{B} 3$ & 23 & $144.1 \pm 118.7$ & & 1 & \\
\hline \multirow{2}{*}{ Masaoka-Koga stage } & I & 23 & $135.8 \pm 119.5$ & 0.356 & 6.215 & $0.238-162.494$ \\
\hline & IIA-IV & 8 & $92.8 \pm 111.6$ & & 1 & \\
\hline \multirow[t]{2}{*}{ Tumor size } & {$[?] 5 \mathrm{~cm}$} & 21 & $128.0 \pm 123.6$ & 0.774 & 0.283 & $0.01-7.879$ \\
\hline & $>5 \mathrm{~cm}$ & 10 & $115.0 \pm 106.9$ & & 1 & \\
\hline \multirow[t]{2}{*}{ Ectopic thymus } & yes & 8 & $132.3 \pm 130.3$ & 0.825 & 3.606 & $0.214-60.735$ \\
\hline & no & 23 & $121.5 \pm 115.6$ & & 1 & \\
\hline
\end{tabular}

Table 5 PD-L2 scores and Multivariate analysis of PD-L2 expression by clinicopathologic characteristics in patients with MG

\section{Discussion}

There was little study investigating the PD-L2 expression in thymoma. And PD-L2 was less well studied. Human PD-L2 are expressed on dendritic cells and medullary thymic epithelial cells ${ }^{[10]}$. High PD-L2 expression may promote tumor metastasis and predict unfavorable prognosis in solid cancer patients after surgery ${ }^{[13]}$. Previous studies showed that PD-L2 exerts its main physiological and pathological function in immune tolerance via dampening and modulating T helper type 2 (Th2) response ${ }^{[14]}$. MG is an autoimmune disease associated with thymus pathologies. A preliminary investigation between the PD-L2 expression in thymoma and MG was shown in the results above.

In this study, the PD-L2 expression was positive in 41 patients (58.6\%). However, Isabelle Rouquette et al found that PD-L2 antibody stained no thymic epithelial tumors ${ }^{[15]}$. Different antibody and cutoff values vary among studies. The positive expression of PD-L2 was associated with B2/B3 histologic type. Because there were few studies put attention in PD-L2 and thymoma, we referred PD-L1 expression. Numerous studies investigating the PD-L1 expression in thymoma indicated that a high expression of PD-L1 was significantly associated with B2/B3 histologic type ${ }^{[16,17]}$. Most studies played attention to the PDL1/2 expression with prognosis. A meta-analysis showed high PD-L2 expression might predict unfavorable prognosis. We did not explore the prognosis in these patients but focus in the PD-L2 expression and MG. 
Patients with B2 histologic type was the most in this study (44.3\%). And the status of MG was associated with B2/B3 histologic types in this study. L. Maggi et al found that AB and B2 thymomas were most frequently observed histologic types in patients with $\mathrm{MG}^{[18]}$. Ströbel, $\mathrm{P}$ et al also observed that B2 was the most frequently ${ }^{[19]}$. However, other authors reported B1 and B2 histologic types as prevalent ${ }^{[20,21]}$. The positive expression of PD-L2 was also associated with a smaller tumor size. What's more, the tumor size was smaller in the patients with MG in this study. This can be explained by earlier diagnosis because of the status of $\mathrm{MG}^{[22]}$. The PD-L2 expression was associated with ectopic thymus in this study. The exist of ectopic thymus may play an important role in the pathogenesis of MG in patients with thymoma because of the TFH cells ${ }^{[6]}$. Furthermore, our group found that ectopic thymus was a poor prognosis factor in patients with $M G{ }^{[23]}$. Not only the Chi square test showed the positive relationship between PD-L2 expression and MG, but also the logistic regression demonstrated a significantly association between PD-L2 expression and MG in the patients with thymoma. Although age was associated with PD-L2 expression with the multivariate logistic regression analysis in the overall patients, but no significantly association was observed in patients with MG. Therefore, we do have sufficient reason to guess that the PD-L2 expression plays a crucial role in the pathogenesis of MG in patients with thymoma.

In this study, a status of MG in these patients with thymoma was associated with B2/B3 histologic types, but has no significantly associated with gender or Masaoka-Koga stage. There was tendency toward younger age in patients with $\mathrm{MG}(\mathrm{p}=0.052)$. Kondo $\mathrm{K}$ et al found that a female dominance in their patients with thymoma and the patients with MG were significantly younger than those without $\mathrm{MG}^{[22]}$. While Jianfei Shen suggested that MG, WHO histology and Masaoka-Koga stage interrelate with one another. The interrelationship drawn from their study was that MG was associated with early clinical stage and WHO histology of AB, B1 and B2-type thymomas ${ }^{[24]}$. The PD-L2 scores was much higher in the patients with MG than without MG (124.1 vs 23.3, $\mathrm{p}<0.001)$. We did an analysis of PD-L2 scores in patients with MG only. We found that there were no significantly difference in PD-L2 scores regarding MGFA stage, Masaoka-Koga stage, tumor size or ectopic thymus, but a tendency toward high PD-L2 scores in patients with B2/B3 types.

The different proportion of T-lymphocytes differing in WHO histologic types may contribute the different prevalence of MG. It is speculated that T-lymphocytes development is involved in the pathogenesis of MG in patients with thymoma ${ }^{[25]}$. And thymomas with $M G$ are enriched for autoreactive $T$ cells with specificity for AChR. And the export of autoreactive CD4+ T cells is of pathogenic relevance of $\mathrm{MG}^{[26]}$. These exporting $\mathrm{T}$ cells may carry special signal in the pathogenesis of MG. And there was a positive association between the percentage of CD4+ T cells and MG severity [4]. Furthermore, PD-1 is highly expressed in TFH, the PD-1 signaling involved decreased GC death and increase TFH cytokine production. PD-1 regulates germinal center B cell survival and the formation and affinity of long-lived plasma cell. ${ }^{[5]}$ Our guess about thymoma related MG pathogenesis is that the PD-L2 co-stimulate PD-1 signal through exporting autoreactive T cells to help germinal center (GC) formation, B cell differentiation into plasma cells and memory cells, and antibody production in secondary lymphoid tissues. Furthermore, we will explore the potential mechanism of PD-L2 signal path in thymoma related MG pathogenesis with the techniques of immunofluorescence and flow cytometry.

However, several limitations associated with present study also warrant mention. First, the number of patients was small. Second, this was a retrospective analysis performed at a single institution. Third, we did not investigate the PD-L2 expression with survive.

In conclusions, a strong expression of PD-L2 in thymoma was significantly associated with thymomatous MG and WHO histologic type B2 and B3. On the basis of our results and analysis, PD-L2 may play a potential role in the pathogenesis of thymomatous MG.

There is no conflict of interest in whichever form at the submission of this manuscript

\section{References}

1. Kazuya Kondo, Kiyoshi Yoshizawa, Masaru Tsuyuguchi, Suguru Kimura, Masayuki Sumitomo, Junji Morita, Takanori Miyoshi, Shoji Sakiyama, Kiyoshi Mukai, Yasumasa Monden. Ann thorac surg 2004(4); 


\section{$77: 1183-8$}

2. Sonia Berrih-Aknin, Rozen Le Panse. Myasthenia Gravis: A Comprehensive Review of Immune Dysregulation and Etiological Mechanisms. J Autoimmun 2014(8): 52, 90-100

3. E Saka, M A Topcuoglu, B Akkaya, A Galati, M Z Onal, A Vincent. Thymus Changes in anti-MuSKpositive and -Negative Myasthenia Gravis. Neurolohy 2005(9):65,782-783

4. E. K. Deenick and C. S. Ma. The regulation and role of T follicular helper cells in immunity. Immunology 2011; 134(4):361-367

5. Kim L Good-Jacobson, Courtney G Szumilas, Lieping Chen, Arlene H Sharpe, Mary M Tomayko, Mark J Shlomchik. PD-1 Regulates Germinal Center B Cell Survival and the Formation and Affinity of Long-Lived Plasma Cells. Nat Immunol. 2010 June; 11(6): 535-542

6. Yang Song, Lei Zhou, Feng Miao, Gang Chen, Yongjun Zhu, Xue Gao, Yiqing Wang, Liewen Pang, Chongbo Zhao, Xiaotian Sun, Zhiming Chen. Increased Frequency of Thymic T Follicular Helper Cells in Myasthenia Gravis Patients with Thymoma. J Thorac Dis 2016;8(3):314-322

7. Min Zhang, Yongan Zhou, Jun Guo, Hongzeng Li, Feng Tian Li Gong, Xianni Wang, Miao Lan, Zhuyi Li, Wei Zhang. Experimental Neurology 2014; 254: 200 -205

8. Hakiri S, Fukui T, Mori S, Kawaguchi K, Nakamura S, Ozeki N, Kato T, Goto M, Yatabe Y, Yokoi K. Clinicopathologic Features of Thymoma With the Expression of Programmed Death Ligand 1. Ann Thorac Surg. 2019 Feb;107(2):418-424.

9.Prerna Guleria, Nuzhat Husain, Saumya Shukla, Sunil Kumar, Rajinder Parshad, Deepali Jain. PD-L1 Immuno-Expression Assay in Thymomas: Study of 84 Cases and Review of Literature. Annals of Diagnostic Pathology 34 (2018) 135-141

10. Julia A Brown, David M Dorfman, Feng-Rong Ma, Elizabeth L Sullivan, Oliver Munoz, Clive R Wood, Edward A Greenfield, Gordon J Freeman. Blockade of Programmed death-1 Ligands on Dendritic Cells Enhances T Cell Activation and Cytokine Production. J Immunol February 1, 2003, 170 (3) 1257-1266

11. Mohammad Reza Zamani, Saeed Aslani, Arash Salmaninejad, Mohammad Reza Javan, Nima Rezaei. PD-1/PD-L and Autoimmunity: A Growing Relationship. Cellular Immunology 310 (2016) 27-41

12. Yokoi K, Kondo K, Fujimoto K. JLCS medical practice guidelines for thymic tumors: summary of recommendations. Jpn J Clin Oncol 2017; 47:1119-22.

13. Huayu Yang, Xiaoxiang Zhou, Lejia Sun, Yilei Mao. Correlation Between PD-L2 Expression and Clinical Outcome in Solid Cancer Patients: A Meta-Analysis. Front oncol.2019;9:47

14. Kenji Ishiwata, Naohiro Watanabe, Miao Guo, Kei Tomihara, Michael J Brumlik, Hideo Yagita, Drew Pardoll, Lieping Chen, Tahiro Shin. Costimulator B7-DC Attenuates Strong Th2 Responses Induced by Nippostrongylus Brasiliensis. J Immunol. (2010) 184:2086-94

15. Isabelle Rouquette, Estelle Taranchon-Clermont, Julia Gilhodes, Maria-Virginia Bluthgen, Romain Perallon, Lara Chalabreysse, Anne De Muret, Veronique Hofman, Alexander Marx, Marie Parrens, Veronique Secq, Vincent Thomas de Montpreville, Françoise Galateau-Salle, Pierre Brousset, Julie Milia, Nicolas Girard, Benjamin Besse, Thierry Jo Molina, Julien Mazières. Immune Biomarkers in Thymic Epithelial Tumors: Expression Patterns, Prognostic Value and Comparison of Diagnostic Tests for PD-L1. Biomark Res. 2019; $7: 28$

16. Sukhmani K Padda, Jonathan W Riess, Erich J Schwartz, Lu Tian, Holbrook E Kohrt, Joel W Neal, Robert B West, Heather A Wakelee. Diffuse High Intensity PD-L1 Staining in Thymic Epithelial Tumors. J thorac oncol 2015; 10:500-8 
17. Marcello Tiseo, Angela Damato, Lucia Longo, Fausto Barbieri, Federica Bertolini, Alessandro Stefani, Mario Migaldi, Letizia Gnetti, Roberta Camisa, Paola Bordi, Sebastiano Buti, Giulio Rossi.Analysis of a Panel of Druggable Gene Mutations and of ALK and PD-L1 Expression in a Series of Thymic Epithelial Tumors (TETs). Lung cancer 2017; 104:24-30

18. Lorenzo Maggi, Francesca Andreetta, Carlo Antozzi, Fulvio Baggi, Pia Bernasconi, Paola Cavalcante, Ferdinando Cornelio, Giuseppe Muscolino, Lorenzo Novellino, Renato Mantegazza. Thymoma-associated Myasthenia Gravis: Outcome, Clinical and Pathological Correlations in 197 Patients on a 20-year Experience. Journal of Neuroimmunology 201-202 (2008) 237-244

19. Philipp Ströbel, Andrea Bauer, Bernhard Puppe, Til Kraushaar, Axel Krein, Klaus Toyka, Ralf Gold, Michael Semik, Reinhard Kiefer, Wilfred Nix, Berthold Schalke, Hans Konrad Müller-Hermelink, Alexander Marx. Tumor Recurrence and Survival in Patients Treated for Thymomas and Thymic Squamous Cell Carcinomas: A Retrospective Analysis. J. Clin. Oncol. 22,1501-1509

20. Evoli, A., Minisci, C., Di Schino, C., Marsili, F., Punzi, C., Batocchi, A.P., Tonali, P.A., Doglietto, G.B., Granone, P., Trodella, L., Cassano, A., Lauriola, L. Thymoma in patients with MG: characteristics and longterm outcome. Neurology 2002;59, 1844-1850

21. Okumura, M., Ohta,M., Tateyama,H.,Nakagawa,K.,Matsumura,A.,Maeda,H., Tada, H., Eimoto, T., Matsuda, H., Masaoka, A.. The World Health Organization histologic classification system reflects the oncologic behavior of thymoma: a clinical study of 273 patients. Cancer 2002; 94, 624-632

22. Kazuya Kondo, Yasumasa Monden. Thymoma and Myasthenia Gravis: A Clinical Study of 1,089 Patients From Japan. Ann Thorac Surg. 2005 Jan;79(1):219-24

23. Jianyong Zou, Chunhua Su, Xueping Lun, Weibing Liu, Weiling Yang, Beilong Zhong, Haoshuai Zhu, Yiyan Lei, Honghe Luo, Zhenguang Chen. Preoperative Anxiety in Patients With Myasthenia Gravis and Risk for Myasthenic Crisis After Extended Transsternal Thymectomy: A CONSORT Study. Medicine (Baltimore). 2016 Mar;95(10):e2828

24. Jianfei Shen, Hongtao Tie, Anyi Xu, Dan Chen, Dehua Ma, Bo Zhang, Chengchu Zhu, Qingchen Wu. Inter-relationship Among Myasthenia Gravis, WHO Histology, and Masaoka Clinical Stage and Effect on Surgical Methods in Patients With Thymoma: A Retrospective Cohort Study. J Thorac Dis. 2018 May;10(5):2981-2990

25. Enrico Ruffini, Pier Luigi Filosso, Claudio Mossetti, Maria Cristina Bruna, Domenico Novero, Patrizia Lista, Caterina Casadio, Alberto Oliaro.Thymoma: Inter-Relationships Among World Health Organization Histology, Masaoka Staging and Myasthenia Gravis and Their Independent Prognostic Significance: A SingleCentre Experience. Eur J Cardiothorac Surg 2011; 40:146-53

26. Alexander Marx , Hans Konrad Müller-Hermelink, Philipp Ströbel. The Role of Thymomas in the Development of Myasthenia Gravis. Ann.N.Y.acad.sci. 2003 998:223-236 